\title{
Outcomes of intraoperative extracorporeal membrane oxygenation versus cardiopulmonary bypass for lung transplantation
}

\author{
Tiago N. Machuca, MD, Stephane Collaud, MD, MSc, Olaf Mercier, MD, PhD, Maureen Cheung, MD, \\ Valerie Cunningham, CCP, S. Joseph Kim, MD, PhD, Sassan Azad, CRA, Lianne Singer, MD, MSc, \\ Kazuhiro Yasufuku, MD, PhD, Marc de Perrot, MD, MSc, Andrew Pierre, MD, MSc, Karen McRae, MD, \\ Thomas K. Waddell, MD, PhD, Shaf Keshavjee, MD, MSc, and Marcelo Cypel, MD, MSc
}

\begin{abstract}
Objective: The study objective was to compare the outcomes of intraoperative extracorporeal membrane oxygenation versus cardiopulmonary bypass support in lung transplantation.

Methods: We performed a retrospective cohort study from a prospective database of adult lung transplantations performed at the University of Toronto from 2007 to 2013. Among 673 lung transplantations performed in the study period, $267(39.7 \%)$ required cardiopulmonary support. There were 39 cases of extracorporeal membrane oxygenation (2012-2013) and 228 cases of cardiopulmonary bypass (2007-2013). Patients who were bridged with extracorporeal life support, underwent a concomitant cardiac procedure, received a combined liver or heart transplant, were colonized with Burkholderia cenocepacia, or required emergency cannulation for cardiopulmonary support were excluded. Finally, 33 extracorporeal membrane oxygenation cases were matched with 66 cases of cardiopulmonary bypass according to age ( \pm 10 years), lung transplantation indication, and procedure type (bilateral vs single lung transplantation).
\end{abstract}

\begin{abstract}
Results: Recipient factors such as body mass index and gender were not different between extracorporeal membrane oxygenation and cardiopulmonary bypass groups. Furthermore, donor variables were similar, including age, body mass index, last $\mathrm{PaO} 2 / \mathrm{FiO} 2$ ratio, smoking history, positive airway cultures, and donor type (brain death and donation after cardiac death). Early outcomes, such as mechanical ventilation requirement, length of intensive care unit stay, and length of hospital stay, significantly favored extracorporeal membrane oxygenation (median $3 \mathrm{vs}$ 7.5 days, $P=.005 ; 5$ vs 9.5 days, $P=.026 ; 19$ vs 27 days, $P=.029$, respectively). Perioperative blood product transfusion requirement was lower in the extracorporeal membrane oxygenation group. The 90-day mortality for the extracorporeal membrane oxygenation group was $6 \%$ versus $15 \%$ for cardiopulmonary bypass $(P=.32)$.
\end{abstract}

Conclusions: Extracorporeal membrane oxygenation may be considered as the first choice of intraoperative cardiorespiratory support for lung transplantation. (J Thorac Cardiovasc Surg 2015;149:1152-7)

See related commentary on pages 1158-60.

Cardiopulmonary bypass $(\mathrm{CPB})$ is the standard and most familiar modality used for intraoperative cardiorespiratory support during lung transplantation (LTx). In most instances $\mathrm{CPB}$ is instituted on a selective basis, although there are programs that use it routinely. ${ }^{1}$ The theoretic

\footnotetext{
From the Toronto Lung Transplant Program, University Health Network, University of Toronto, Toronto, Ontario, Canada.

TNM is supported by a Research Fellowship from the American Society of Transplantation.

Disclosures: S. Joseph Kim reports consulting and lecture fees from Astellas-Pharma. All other authors have nothing to disclose with regard to commercial support.

Received for publication Aug 4, 2014; revisions received Nov 11, 2014; accepted for publication Nov 18, 2014; available ahead of print Jan 10, 2015.

Address for reprints: Marcelo Cypel, MD, MSc, Toronto Lung Transplant Program, Toronto General Hospital, 200 Elizabeth St, 9N946, Toronto, Ontario, M5G 2C4 Canada (E-mail: marcelo.cypel@uhn.ca).

0022-5223/ $\$ 36.00$

Copyright (c) 2015 by The American Association for Thoracic Surgery

http://dx.doi.org/10.1016/j.jtcvs.2014.11.039
}

benefit of providing controlled low-pressure reperfusion is obviously counterbalanced by the inflammatory response leading to coagulopathy, neutrophil activation, and complement activation that ultimately lead to endorgan injury. ${ }^{2}$ Furthermore, the use of CPB has been highlighted as an independent factor associated with primary graft dysfunction. ${ }^{3}$

In theory, the advantages of extracorporeal membrane oxygenation (ECMO) are a relatively miniaturized circuit that requires lower priming volumes and the lack of both air-blood contact and cardiotomy suction, leading to lesser anticoagulation requirement and potentially to lesser coagulopathy and systemic inflammatory response. In cardiac surgery procedures, the use of a miniaturized CPB circuit has been shown to be associated with lower transfusion requirement, a reduction in peak troponin, and a lower incidence of neurologic damage in comparison with conventional CPB. ${ }^{4}$ In a more recent meta-analysis, miniaturized circuits have been shown to have decreased inflammatory response (measured by polymorphonuclear elastase), lesser hemodilution, lesser need for inotropic 


$$
\begin{aligned}
& \text { Abbreviations and Acronyms } \\
& \begin{aligned}
\text { ACT } & =\text { activated clotting time } \\
\text { CPB } & =\text { cardiopulmonary bypass } \\
\text { ECLS } & =\text { extracorporeal life support } \\
\text { ECMO } & =\text { extracorporeal membrane oxygenation } \\
\text { ICU } & =\text { intensive care unit } \\
\text { LTx } & =\text { lung transplantation } \\
\text { pRBC } & =\text { packed red blood cells }
\end{aligned}
\end{aligned}
$$

support, lower peak creatinine level, lesser need for red blood cell transfusion, and lower incidence of atrial fibrillation. These advantages were reflected in a lower requirement for mechanical ventilation, shorter intensive care unit (ICU) and hospital stay, and ultimately lower hospital mortality. ${ }^{5}$

With all of these findings taken together, one might speculate that ECMO could be a better option for intraoperative support in LTx, an operation that is already marked by a heightened inflammatory milieu. With increasing use of ECMO as a bridge to transplant ${ }^{6-12}$ or to recovery in cases of severe primary graft dysfunction, ${ }^{13-17}$ some programs have pioneered and expanded its use for the intraoperative period of LTx. ${ }^{18}$ Nevertheless, this approach is still underused, and there are only few retrospective reports in the literature addressing this issue. ${ }^{19}$

On the basis of our growing extracorporeal life support (ECLS) experience, in 2012 we started to use venoarterial ECMO instead of CPB for intraoperative support in LTx. In the present study, we compared the outcomes of LTx using ECMO versus CPB using a matched cohort design to reduce the potential for confounding by indication.

\section{MATERIALS AND METHODS}

We conducted a retrospective cohort study using a prospective database of patients who underwent LTx from January 2007 to December 2013 and required intraoperative cardiopulmonary support. Cases were labeled as ECMO or CPB and were matched on a 1:2 ratio according to transplant indication, single or double LTx, and age. The latter was frequency matched within a 10-year window. To further reduce the risk of biased inferences, we excluded from the cohort patients who (1) were bridged with ECLS, (2) underwent concomitant cardiac procedure or combined liver or heart transplantation, (3) were colonized with Burkholderia cenocepacia, or (4) required emergency cannulation for cardiopulmonary support. Emergency cannulation was defined as institution of CPB because of severe instability during anesthesia induction or massive bleeding. This study was approved by the University Health Network Research Ethics Board.

It has been our practice to use cardiopulmonary support on a selective basis. At the time of the first lung implant, we would institute support in cases of hemodynamic instability or inability to tolerate pulmonary artery clamping or single lung ventilation. In the event that the first lung is implanted without difficulty, then cardiopulmonary support is subsequently initiated if hemodynamic instability or inadequate gas exchange ensues when attempting to support the patient on the newly implanted initial graft. Patients with primary pulmonary hypertension routinely are placed on mechanical support at the initiation of surgery.
The patient population is summarized in Figure 1, A. Of 673 LTxs, $39.7 \%$ (267) were performed with intraoperative cardiopulmonary support. After the specific exclusion criteria were applied, 33 ECMO cases were matched with $66 \mathrm{CPB}$ cases. The number of cases performed by year, with the correspondent intraoperative support (none, $\mathrm{CPB}$, or ECMO) is shown in Figure 1, B. Intraoperative ECMO was first used in 2012 and became the most common mode of intraoperative cardiopulmonary support for LTx within the subsequent year.

Donor and recipient baseline characteristics are shown in Table 1. Donor demographics were similar between groups in terms of age, positive smoking history, and positive bronchoalveolar lavage culture (Table 1). There was a trend to more male donors and more ex vivo lung perfusion use in the ECMO group.

Matching of the recipients was successfully performed according to indication (54\% of idiopathic pulmonary fibrosis), type of transplant ( $85 \%$ double lung in CPB vs $82 \%$ in ECMO), and age (mean $53.4 \pm$ 13.5 years for $\mathrm{CPB}$ vs $55.8 \pm 12.5$ years for ECMO). Gender and body mass index were not different between groups.

\section{Cardiopulmonary Bypass Technique}

The traditional CPB system consists of a (1) Stockert S III console (Stockert, Munich, Germany); (2) Medtronic Carmeda BioActive Surface closed venous reservoir (Medtronic Inc, Minneapolis, Minn); (3) Sorin Revolution centrifugal pump (Sorin Group, Mirandola, Italy); (4) Maquet Quadrox-I Adult Softline coated oxygenator (Maquet Cardiopulmonary, Rastatt, Germany) (rated flow 0.5-7.0 L/min, $1.8 \mathrm{~m}^{2}$ microporous polypropylene gas fiber membrane surface, low prime volume of 215 $\mathrm{mL}, 0.4 \mathrm{~m}^{2}$ polyurethane heat exchanger surface area) with a (5) Sorin Physio Micro 27 arterial filter and (6) Sorin Physio tubing pack. Prime volume is minimized to $1.2 \mathrm{~L}$ crystalloid (Ringer's lactate). Perfusion technique routinely included a retrograde autologous prime after cannulation when stable hemodynamics permitted, to minimize the circuit crystalloid to $800 \mathrm{~mL}$. Before cannulation, patients were fully heparinized and $\mathrm{CPB}$ was instituted with an activated clotting time (ACT) greater than 480 seconds.

\section{Extracorporeal Membrane Oxygenation Technique}

The ECLS circuit consisted of (1) a Bio-Medicus centrifugal pump (Medtronic Inc); (2) a Maquet Quadrox-I Adult Softline coated oxygenator (Maquet Cardiopulmonary) (rated flow 0.5-7.0 L/min, $1.8 \mathrm{~m}^{2}$ microporous polypropylene gas fiber membrane surface, low prime volume of $215 \mathrm{~mL}$, $0.4 \mathrm{~m}^{2}$ polyurethane heat exchanger surface area); and (3) a Medtronic Carmeda BioActive Surface Tubing (Medtronic Inc). In an effort to optimize patient safety, minimize circuit surface area, and decrease the risk of air entrainment, the circuit was simplified. The bridge was removed, as were pressure isolators and sampling manifolds. The ECLS circuit prime total volume was $800 \mathrm{~mL}$. Before cannulation, patients received $5000 \mathrm{IU}$ of intravenous unfractionated heparin. If an ACT greater than 200 seconds was not achieved, additional heparin was administered to achieve a target ACT of 180 to 200 seconds. ACT is measured every 15 to 30 minutes during the procedure.

\section{Arterial Cannulation}

The cannulation sites were selected primarily on the basis of surgeon preference and the type of procedure (single vs double lung transplant). In the cases of central (aortic) cannulation, a 20F to 22F Medtronic cannula was used, according to patient size and predicted cardiac output. In the cases of femoral artery cannulation, $17 \mathrm{~F}$ to $20 \mathrm{~F}$ Medtronic cannulae were used using a percutaneous Seldinger, open Seldinger, or cut-down technique. Central cannulation was used in $84 \%$ of cases.

\section{Venous Cannulation}

Likewise, cannulation sites and type of cannulae were selected on the basis of surgeon preference and transplant type. In cases of central 

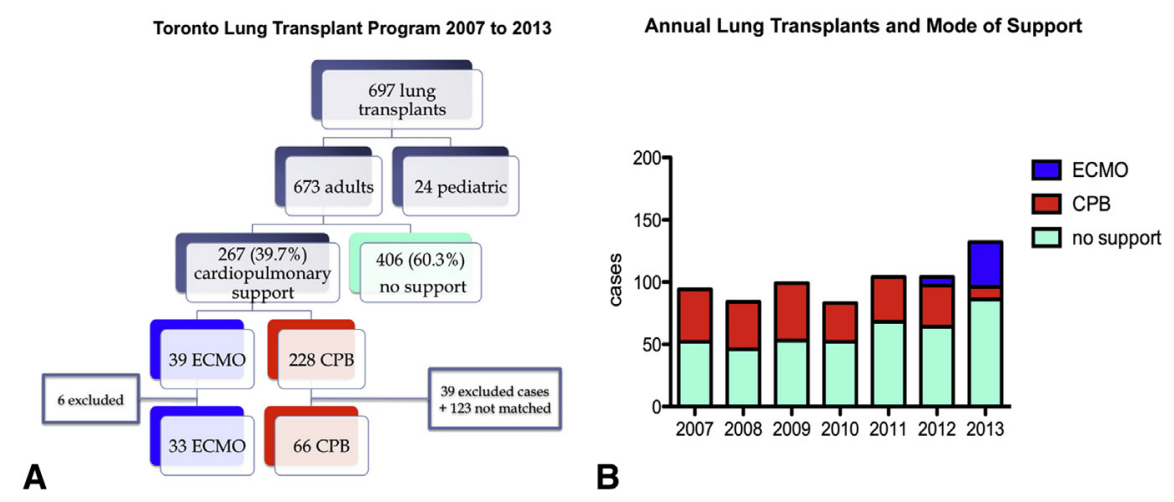

FIGURE 1. A, Study flow diagram. Patients excluded were those who required bridge with ECLS, who underwent concomitant cardiac procedure or combined liver or heart transplantation, who were colonized with Burkholderia cenocepacia, or who required emergency cannulation. B, Number of lung transplants performed annually, depicting the intraoperative mode of support used: none, ECMO, or CPB. CPB, Cardiopulmonary bypass; ECMO, extracorporeal membrane oxygenation.

cannulation, cannulae usually used were $32 \mathrm{~F}$ to $36 \mathrm{~F}$ single-staged Medtronic cannula or $34 \mathrm{~F}$ to $46 \mathrm{~F}$ double-staged Sarnes cannula. For cases of femoral vein cannulation, a long $21 \mathrm{~F}$ or $25 \mathrm{~F}$ Medtronic cannula was inserted with a Seldinger technique. Peripheral cannulation (femoral vein) was used in $60 \%$ of cases.

\section{Statistical Analysis}

Categoric variables were compared using the Fisher exact test. For continuous variables, comparisons were performed using the Student $t$ test or Mann-Whitney test, whenever appropriate. Survival curves were plotted using the Kaplan-Meier product limit method and compared with the log-rank test. We used GraphPad Prism software (GraphPad Software Inc, San Diego, Calif) to conduct all statistical analyses.

TABLE 1. Donor and recipient baseline demographics

\begin{tabular}{lccl}
\hline \multicolumn{1}{c}{ Variable } & CPB $(\mathbf{n}=\mathbf{6 6})$ & ECMO $(\mathbf{n}=\mathbf{3 3})$ & $\boldsymbol{P}$ value \\
\hline Donor variables & & & \\
Age (y) & $44.7 \pm 16$ & $41.8 \pm 19.8$ & .42 \\
Gender (male) & $28(42 \%)$ & $21(63 \%)$ & .056 \\
Smoking & $37(56 \%)$ & $15(45 \%)$ & .39 \\
Positive BAL & $45(68 \%)$ & $17(51 \%)$ & .12 \\
EVLP & $8(12 \%)$ & $9(27 \%)$ & .08 \\
Recipient variables & & & \\
Indication & & & 1 \\
IPF & $36(54 \%)$ & $18(54 \%)$ & \\
Cystic fibrosis & $10(15 \%)$ & $5(15 \%)$ & \\
Emphysema & $6(9 \%)$ & $3(9 \%)$ & \\
Scleroderma & $6(9 \%)$ & $3(9 \%)$ & \\
Retransplant & $4(6 \%)$ & $2(6 \%)$ & \\
Others & $4(6 \%)$ & $2(6 \%)$ & \\
Double lung & $56(85 \%)$ & $27(82 \%)$ & .77 \\
Age (y) & $53.4 \pm 13.5$ & $55.8 \pm 12.5$ & .77 \\
Gender (male) & $41(62 \%)$ & $19(57 \%)$ & .66 \\
BMI & $24 \pm 4$ & $23 \pm 4$ & .67 \\
\hline
\end{tabular}

Parametric continuous variables are expressed as mean \pm standard deviation and were compared with the Student $t$ test. Categoric variables were compared with the Fisher exact test. $B A L$, Bronchoalveolar lavage; $B M I$, body mass index; $C P B$, cardiopulmonary bypass; $E C M O$, extracorporeal membrane oxygenation; $E V L P$, ex vivo lung perfusion; $I P F$, idiopathic pulmonary fibrosis. Others include primary pulmonary arterial hypertension and lymphangioleiomyomatosis.

\section{RESULTS}

Intraoperative pump times were similar for $\mathrm{CPB}$ and ECMO $(199 \pm 90$ minutes vs $210 \pm 84$ minutes, respectively). The warm ischemic times (implant times) did not differ between groups for both double and single LTxs (Table 2).

The intraoperative requirement of blood products significantly favored the ECMO group. There was less packed red blood cell (pRBC) transfusion (median 3 vs 6 units; $P<.001$ ), less platelet ( 0 vs 1 adult pooled units; $P=.007$ ), and less fresh-frozen plasma (0 vs 4 units; $P=.006)$ requirements compared with the CPB group. The transfusion requirement for pRBC over the first 72 hours after transplant also favored the ECMO group (1 vs 2 units; $P=.014$ ).

With regard to early post-transplant outcomes, patients in the ECMO group had a shorter time on the ventilator (median 3 vs 7.5 days; $P=.005$ ), shorter ICU length of stay (5 vs 9.5 days; $P=.026$ ), and shorter hospital length of stay (19 vs 27 days; $P=.029$ ) (Table 2). Although 5 patients required postoperative ECMO in the $\mathrm{CPB}$ group and no patient required it in the ECMO group, this difference was not statistically significant. Twelve patients $(18 \%)$ required postoperative dialysis in the CPB group versus $3(9 \%)$ in the ECMO group $(P=.37)$. In the CPB group, 18 patients $(27 \%)$ required reoperation for bleeding, whereas only 3 patients $(9 \%)$ did so in the ECMO group $(P=.04)$.

The 12-month Kaplan-Meier survival curves were not different between the groups (Figure 2). The 90-day mortality was not significantly different between the groups ( $6 \% \mathrm{ECMO}$ vs $15 \% \mathrm{CPB}, P=.32$ ). Causes of mortality are summarized in Table 3. Of note, the stroke early mortality in the ECMO group was a 41-year-old woman with familial leukodystrophy and pulmonary fibrosis who underwent an uneventful double-lung transplant on venoarterial ECMO. After recovering from medical complications 
TABLE 2. Intraoperative data and early post-transplant outcomes

\begin{tabular}{lccc}
\hline \multicolumn{1}{c}{ Variable } & $\begin{array}{c}\text { CPB } \\
(\mathbf{n = 6 6 )}\end{array}$ & $\begin{array}{c}\text { ECMO } \\
(\mathbf{n = 3 3 )}\end{array}$ & $\begin{array}{c}\boldsymbol{P} \\
\text { value }\end{array}$ \\
\hline Intraoperative & & & \\
Pump time (min) & $199 \pm 90$ & $210 \pm 84$ & .38 \\
Warm ischemic time L (min) & $69 \pm 17$ & $73 \pm 23$ & .37 \\
Warm ischemic time R (min) & $64 \pm 14$ & $70 \pm 20$ & .123 \\
Warm ischemic time SLTx (min) & $74 \pm 9$ & $74 \pm 19$ & .94 \\
Blood transfusion & & & \\
$\quad$ pRBCs (units) & $6(3-10)$ & $3(2-5.5)$ & $<.001$ \\
$\quad$ Platelets (units) & $1(0-2)$ & $0(0-0.5)$ & .007 \\
$\quad$ FFP (units) & $4(1.7-7.2)$ & $0(0-0.5)$ & .006 \\
Postoperative & & & \\
pRBC up to 72 h (units) & $2(1-6)$ & $1(0.5-3)$ & .014 \\
Length of MV (d) & $7.5(2-18)$ & $3(2.5-5)$ & .005 \\
ICU stay (d) & $9.5(3-21)$ & $5(3-9)$ & .026 \\
Hospital stay & $27(17-42)$ & $19(14-30)$ & .029 \\
ECLS postoperative requirement & $5(7.5 \%)$ & 0 & .166 \\
Dialysis requirement & $12(18 \%)$ & $3(9 \%)$ & .37 \\
Reoperation (bleeding) & $18(27 \%)$ & $3(9 \%)$ & .04 \\
90-d mortality & $10(15 \%)$ & $2(6 \%)$ & .32 \\
\hline
\end{tabular}

Parametric continuous variables are expressed as mean \pm standard deviation and were compared with Student $t$ test. Nonparametric continuous variables are expressed as median (25\%-75\% interquartile range) and were compared with the Mann-Whitney test. Categoric variables were compared with the Fisher exact test. $C P B$, Cardiopulmonary bypass; $E C L S$, extracorporeal life support; $E C M O$, extracorporeal membrane oxygenation; $F F P$, fresh-frozen plasma; $I C U$, intensive care unit; $M V$, mechanical ventilation; $p R B C$, packed red blood cells; SLTx, single lung transplant; $L$, left; $R$, right. Warm ischemic time corresponded to the interval from graft placement inside the thoracic cavity until pulmonary artery reperfusion.

(acute antibody-mediated rejection), she was about to be discharged when she had a massive intracranial hemorrhage 60 days after transplantation.

\section{DISCUSSION}

With increasing experience gained in our ECLS program with both bridge to transplant and bridge to recovery situations, we have increasingly used venoarterial ECMO

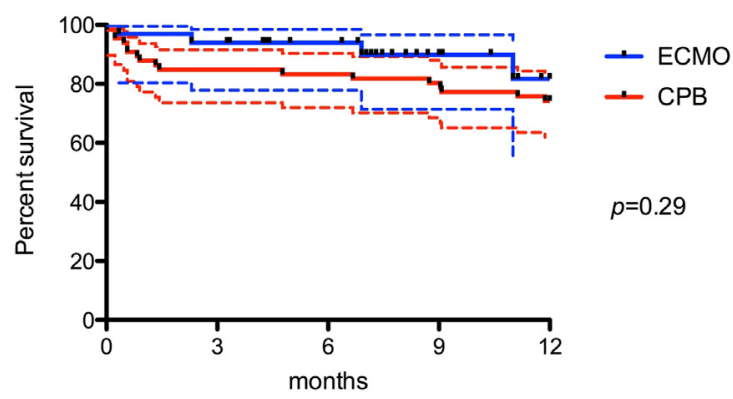

Patients at risk:

$\begin{array}{lllllc}\text { ECMO } & 33 & 32 & 26 & 14 & 8 \\ \text { CPB } & 66 & 57 & 56 & 54 & 49\end{array}$

FIGURE 2. Kaplan-Meier survival curves and $95 \%$ confidence interval limits for 12 months. Although there is a decrease in the CPB curve during the first 2 months, there is no statistically significant difference $(P=.29)$. $C P B$, Cardiopulmonary bypass; ECMO, extracorporeal membrane oxygenation. instead of standard CPB for patients requiring intraoperative support during LTx. In our experience, the theoretic advantages of the ECMO circuit and technique appear to be borne out: Patients undergoing LTx who were supported intraoperatively with ECMO demonstrated less requirement for mechanical ventilation, had shortened ICU and hospital length of stay, and required less blood product transfusions during the perioperative period when compared with matched recipients who were supported with conventional CPB.

Given the results presented, ECMO has become our preferred mode of support for LTx cases requiring cardiopulmonary support for intraoperative respiratory or hemodynamic instability. It is important to note that for implementation of intraoperative ECMO, important modifications are used to prevent complications of air embolism (because there is no venous reservoir to trap significant entrained air): (1) For right atrial cannulation, 2 purse strings are used and an umbilical tape is also snared around the cannula; (2) the circuit includes a centrifugal pump with a lock mechanism in case of air entrainment; (3) the oxygenator used is a Quadrox with de-airing ports; and (4) an emergency CPB circuit as a backup is always available in our operating room in the eventuality of any problem with the ECMO circuit. Because the majority of patients undergoing LTx require only partial or subtotal cardiopulmonary support and stabilize with partial flows compared with patients undergoing cardiac surgery, the heart is generally less empty, which again decreases the likelihood of air embolism. We did not have problems with air in the circuit in any of our intraoperative ECMO cases to date. Even in an event of that occurring, the risk of air passing beyond the new-generation oxygenators is minimal.

The current report demonstrated how surgical advances and evolution of practice can improve outcomes of LTx in an experienced large-volume center. Our results corroborate the previous findings of the Vienna and Hannover LTx programs. In the former report, intraoperative ECMO was used in 130 patients, with 51 of them being maintained postoperatively with elective ECMO, versus 27 patients who underwent LTx with CPB (mainly to concomitantly correct cardiac defects). ${ }^{18}$ Although Kaplan-Meier survival curves did not differ, patients in the intraoperative ECMO groups presented a trend toward lesser ICU stay and had significantly shorter hospital stay when compared with CPB. In the latter study, 46 LTxs with intraoperative ECMO cases were compared with 46 intraoperative CPB cases. ${ }^{19}$ Patients undergoing $\mathrm{CPB}$ required more pRBC and platelet transfusions intraoperatively, presented with higher requirements for dialysis and new ECMO implantation, and, more important, had a higher inhospital mortality $(39 \%$ vs $13 \%)$. No differences were found with regard to time on mechanical ventilation and 
TABLE 3. Causes of 90-day mortality with respective recipient demographics

\begin{tabular}{lcclrl}
\hline \multicolumn{3}{c}{ Age (y)/ } & \multicolumn{5}{c}{ Type of } & Survival \\
Group & gender & Diagnosis & transplantation & (d) & Cause of death \\
\hline ECMO & $69 / \mathrm{F}$ & COPD & DLTx & 10 & Sepsis \\
ECMO & $41 / \mathrm{F}$ & IPF & DLTx & 69 & Stroke \\
CPB & $61 / \mathrm{M}$ & IPF & DLTx & 25 & Sepsis \\
CPB & $44 / F$ & Redo & DLTx & 27 & Sepsis \\
CPB & $69 / \mathrm{M}$ & IPF & DLTx & 17 & Retroperitoneal \\
& & & & & bleeding \\
CPB & $58 / F$ & IPF & DLTx & 7 & Sepsis \\
CPB & $75 / M$ & IPF & DLTx & 7 & Stroke \\
CPB & $58 / F$ & IPF & DLTx & 14 & Hemorrhage \\
CPB & $69 / M$ & COPD & DLTx & 43 & Sepsis \\
CPB & $50 / M$ & Redo & DLTx & 1 & Hemorrhage \\
CPB & $67 / M$ & IPF & SLTx & 17 & Stroke \\
CPB & $64 / M$ & IPF & SLTx & 40 & Status epilepticus \\
\hline COPD, & Ch & &
\end{tabular}

COPD, Chronic obstructive pulmonary disease; $C P B$, cardiopulmonary bypass; $D L T x$, double lung transplant; $E C M O$, extracorporeal membrane oxygenation; $F$, female; IPF, idiopathic pulmonary fibrosis; $M$, male; Redo, retransplantation; SLTX, single lung transplant.

ICU stay, although one should note that while $98 \%$ of CPB cases were weaned intraoperatively, this scenario only occurred for 59\% of ECMO cases because a high proportion of patients had pulmonary hypertension as the primary diagnosis.

Although these reports have shown the benefits of ECMO comparing all ECMO with all CPB cases (without a matched design), we opted to study more comparable populations by excluding patients with conditions that may increase the risk of estimating a biased treatment effect. Thus, patients bridged with ECLS, patients with combined lung-liver or heart-lung transplants, recipients undergoing concomitant cardiac procedures, pediatric patients, and those with Burkholderia cenocepacia colonization were excluded from this analysis. Moreover, we also excluded those patients who underwent emergency cannulation, because in these cases an intraoperative complication probably occurred and they were more likely to be supported with $\mathrm{CPB}$, a situation that could bias (worsen) the outcomes of this group. In the previous reports, these populations were not excluded from the analysis. Also of note is the fact that previous studies evaluated younger populations (mean age of 42 and 42.8 years vs 55.8 years in our study) probably because of a higher prevalence of patients with primary pulmonary arterial hypertension in detriment of the idiopathic pulmonary fibrosis cohort. As shown in Table 1 , more than $50 \%$ of our supported cases were patients with idiopathic pulmonary fibrosis, which is currently a predominant patient population receiving LTx in North America. Last, both of these centers routinely used planned post-transplant ECMO for the patients with primary pulmonary hypertension. We did not use elective postoperative ECMO in any of our patients.
Although the ECMO population in our series was more recent than some of the CPB population, the span of the study was contemporary and relatively short (7 years). Furthermore, in this period we did not implement any major programmatic change in clinical practice (ie, immunosuppressive regimen, antimicrobial prophylaxis, or post-transplant follow-up). To further reassure against a potential temporal bias, a comparison of our program's survival outcomes in patients not requiring cardiopulmonary support who received transplantations between 2007 and 2011 (contemporary to most of the CPB cases included) versus between 2012 and 2013 (contemporary to the ECMO cases) did not show any significant difference (data not shown). As previously mentioned, in accordance with our more recent practice, the ECMO group tended to include more donor lungs that underwent ex vivo lung perfusion $(P=.08)$. Nevertheless, we have previously shown that despite being higher risk donor lungs, once submitted to ex vivo lung perfusion they present similar outcomes to standard donors. $^{20,21}$

\section{Study Limitations}

Limitations from our study reside in its single-center, retrospective nature and the potential for temporal bias as addressed earlier. The matched design was used to reduce the potential for confounding bias that was noted to be present in previous studies.

\section{CONCLUSIONS}

In our experience, ECMO has shown significant advantages over $\mathrm{CPB}$ for both intraoperative and early post-transplant outcomes. Patients undergoing LTx who were supported intraoperatively with ECMO demonstrated a shorter duration of mechanical ventilation, had shorter ICU and hospital length of stay, and required less blood products during the perioperative period when compared with matched recipients who were supported with conventional CPB.

These findings suggest that ECMO may be considered to be the preferred choice for intraoperative cardiopulmonary support for LTx. Conventional CPB will certainly remain an essential support tool for patients undergoing LTx and concomitant cardiac repair procedures, cases of complex atrial anastomosis requiring heart arrest, and in emergency situations such as massive intraoperative bleeding.

\footnotetext{
References

1. Marczin N, Royston D, Yacoub M. Pro: lung transplantation should be routinely performed with cardiopulmonary bypass. J Cardiothorac Vasc Anesth. 2000;14: 739-45.

2. McRae K. Con: lung transplantation should not be routinely performed with cardiopulmonary bypass. J Cardiothorac Vasc Anesth. 2000;14:746-50.

3. Diamond JM, Lee JC, Kawut SM, Shah RJ, Localio AR, Bellamy SL, et al. Clinical risk factors for primary graft dysfunction after lung transplantation. Am J Respir Crit Care Med. 2013;187:527-34.
} 
4. Zangrillo A, Garozzo FA, Biondi-Zoccai G, Pappalardo F, Monaco F, Crivellari M, et al. Miniaturized cardiopulmonary bypass improves short-term outcome in cardiac surgery: a meta-analysis of randomized controlled studies. J Thorac Cardiovasc Surg. 2010;139:1162-9.

5. Anastasiadis K, Antonitsis $\mathrm{P}$, Haidich AB, Argiriadou H, Deliopoulos A, Papakonstantinou $\mathrm{C}$, et al. Use of minimal extracorporeal circulation improves outcome after heart surgery; a systematic review and meta-analysis of randomized controlled trials. Int J Cardiol. 2013;164:158-69.

6. Fuehner T, Kuehn C, Hadem J, Wiesner O, Gottlieb J, Tudorache I, et al. Extracorporeal membrane oxygenation in awake patients as bridge to lung transplantation. Am J Respir Crit Care Med. 2012;185:763-8.

7. Hoopes CW, Kukreja J, Golden J, Davenport DL, Diaz-Guzman E, Zwischenberger JB. Extracorporeal membrane oxygenation as a bridge to pulmonary transplantation. J Thorac Cardiovasc Surg. 2013;145:862-8.

8. Javidfar J, Brodie D, Iribarne A, Jurado J, Lavelle M, Brenner K, et al. Extracorporeal membrane oxygenation as a bridge to lung transplantation and recovery. J Thorac Cardiovasc Surg. 2012;144:716-21.

9. Lang G, Taghavi S, Aigner C, Renyi-Vamos F, Jaksch P, Augustin V, et al, Primary lung transplantation after bridge with extracorporeal membrane oxygenation: a plea for a shift in our paradigms for indications. Transplantation. 2012; 93:729-36.

10. Toyoda Y, Bhama JK, Shigemura N, Zaldonis D, Pilewski J, Crespo M, et al. Efficacy of extracorporeal membrane oxygenation as a bridge to lung transplantation. J Thorac Cardiovasc Surg. 2013;145:1065-71.

11. Cypel M, Keshavjee S. Extracorporeal life support as a bridge to lung transplantation. Clin Chest Med. 2011;32:245-51.

12. de Perrot M, Granton JT, McRae K, Cypel M, Pierre A, Waddell TK, et al. Impact of extracorporeal life support on outcome in patients with idiopathic pulmonary arterial hypertension awaiting lung transplantation. J Heart Lung Transplant. 2011;30:997-1002.
13. Bermudez CA, Adusumilli PS, McCurry KR, Zaldonis D, Crespo MM Pilewski JM, et al. Extracorporeal membrane oxygenation for primary graft dysfunction after lung transplantation: long-term survival. Ann Thorac Surg. 2009;87:854-60.

14. Fischer S, Bohn D, Rycus P, Pierre AF, de Perrot M, Waddell TK, et al. Extracorporeal membrane oxygenation for primary graft dysfunction after lung transplantation: analysis of the Extracorporeal Life Support Organization (ELSO) registry J Heart Lung Transplant. 2007;26:472-7.

15. Hartwig MG, Walczak R, Lin SS, Davis RD. Improved survival but margina allograft function in patients treated with extracorporeal membrane oxygenation after lung transplantation. Ann Thorac Surg. 2012;93:366-71.

16. Mason DP, Boffa DJ, Murthy SC, Gildea TR, Budev MM, Mehta AC, et al Extended use of extracorporeal membrane oxygenation after lung transplantation. J Thorac Cardiovasc Surg. 2006;132:954-60.

17. Oto T, Rosenfeldt F, Rowland M, Pick A, Rabinov M, Preovolos A, et al. Extracorporeal membrane oxygenation after lung transplantation: evolving technique improves outcomes. Ann Thorac Surg. 2004;78:1230-5.

18. Aigner C, Wisser W, Taghavi S, Lang G, Jaksch P, Czyzewski D, et al. Institutional experience with extracorporeal membrane oxygenation in lung transplantation. Eur J Cardiothorac Surg. 2007;31:468-74.

19. Ius F, Kuehn C, Tudorache I, Sommer W, Avsar M, Boethig D, et al. Lung transplantation on cardiopulmonary support: venoarterial extracorporeal membrane oxygenation outperformed cardiopulmonary bypass. J Thorac Cardiovasc Surg. 2012;144:1510-6.

20. Cypel M, Yeung JC, Liu M, Anraku M, Chen F, Karolak W, et al. Normothermic ex vivo lung perfusion in clinical lung transplantation. N Engl J Med. 2011;364 1431-40.

21. Cypel M, Yeung JC, Machuca T, Chen M, Singer LG, Yasufuku K, et al. Experience with the first 50 ex vivo lung perfusions in clinical transplantation. $J$ Thorac Cardiovasc Surg. 2012;144:1200-6.

Readers who found these articles interesting may also like to read the following papers found in recent and future issues of our sister publications, Seminars in Thoracic and Cardiovascular Surgery and Operative Techniques in Thoracic and Cardiovascular Surgery!

News and Views: Todd Rosengart. The 1000th VAD, The Great Rivalry, and the Grand Experiment of the Texas Medical Center. Semin Thorac Cardiovasc Surg. Spring 2014;26(1):1-3.

News and Views: O. Howard Frazier. Mechanical Circulatory Assist Device Development at the Texas Heart Institute. Semin Thorac Cardiovasc Surg. Spring 2014;26(1):4-13.

State of the Art: Steven Singh. The State of the Art in Corporeal Membrane Oxygenation. Semin Thorac Cardiovasc Surg. Expected publication August 2015.

Carmelo Milano. Heart Transplantation after Left Ventricular Assist Device. Oper Tech Thorac Cardiovasc Surg. Expected publication November 2014.

Mani Daneshmand. Bilateral Sequential Lung Transplantation—evolution to simplify. Oper Tech Thorac Cardiovasc Surg. Summer 2014;19(2):138-151.

Charles Hoopes. Ambulatory Extracorporeal Membrane Oxygenation. Oper Tech Thorac Cardiovasc Surg. Summer 2014;19(2):129137.

Pankaj Saxena. Techniques for lung procurement following donation after circulatory death. Oper Tech Thorac Cardiovasc Surg. Expected publication February 2015.

Ashok Babu. Techniques for venoarterial ECMO support and conversion to temporary left ventricular Assist Device. Oper Tech Thorac Cardiovasc Surg. Expected publication February 2015.

Simon Maltais. Non-sternotomy approaches to left ventricular assist device placement. Oper Tech Thorac Cardiovasc Surg. Expected publication February 2015.

Anelechi Anyanwu. Non-Sternotomy Approaches to Left Ventricular Assist Device Placement: Combined Left Subcostal—Right Mini-Thoracotomy Technique. Oper Tech Thorac Cardiovasc Surg. Expected publication February 2015. 